\title{
Models and Methods to Estimate Digitalization Success Predictively
}

\author{
Alexander S. Geyda \\ St. Petersburg Institute for Informatics and Automation \\ of the Russian Academy of Sciences \\ St. Petersburg, Russia \\ alex19650406@gmail.com
}

\begin{abstract}
The article outlines models of the formation of digitalization effects on the example of modern information (that is - digital) operations use in technological systems and methods to estimate indicators of digitalization success predictively. Models and methods constructed based on graph theory, probability theory and technical systems efficiency theory. Models describe changes in non-information operations as a result of the use of information operations as a reaction to environment change and then - to estimate effects of noninformation operations in changing environments. Such models and methods can be used, for example, to estimate the digitalization performance, efficiency, and effectiveness indicators based on predictive mathematical models of information technology usage. Such models could be used for the estimation of dynamic capability and indicators of system potential as a result of technological systems digitalization. The estimation of mentioned operational properties of digitalization is obtained by plotting the dependences of predicted values of operational properties of the use of information technology as a result of digitalization against the variables and options of the problems to be solved.
\end{abstract}

Keywords-digitalization, effects, performance, efficiency, capabilities, models

\section{INTRODUCTION}

The research on the use of information technologies traditionally implemented based on the operational properties [1-6] of such use. The operational properties of the objects under study are an extensive class of properties of various objects, such that these properties characterize the results of the activity with these objects [7,8]. Therefore, the operational properties form the basis of the quality of objects under research. These properties are manifested at the boundary of the object in which the activity is implemented and in the environment. Operational (or, alternatively, pragmatic, praxeological) properties are estimated by effects (main results) of activity at the boundary, and then - by these effects compliance with the requirements of the environment and goals perceived due to environment demands. Activity is always implemented (by humans or actuators, devices under humans' control) with use of certain information operations. At least such information operations are conducted using the senses or speech. Modern information operations are conducted using digital technologies. Information operations are considered as special elements of activity whose objectives are to obtain information about system and environment states and to generate information about future functioning, but not to exchange matter and energy. As it was mentioned, information operations are always implemented in accordance with certain information technology. Information technology objective is to describe the use of information operations in possible circumstances.

The mechanisms of the formation of activity effects and the subsequent formation of operational properties, considering the use of information operations, especially operations, performed with modern (digital) information technologies (IT), have not been studied in enough detail. Such study shall make it possible to predict the effects of activity with mathematical models, depending on the variable characteristics of the information operations use during system functioning. In this case mathematical problems of evaluation, analysis, and synthesis [8,9] of information technologies use. Unfortunately, there are no suitable models and methods for analytically describing the effects of information operations, considering further use of the effects, produced by information operations. That is why operational properties of information technologies use were not measured analytically, on predictive mathematical models. This, in turn, caused by the absence of a universally accepted concept of causes and effects of information operations, and next - of information operations effects use for material effects change. Particularly, cause and effects relationships are not studied well for non-information (material) effects that are obtained by non-information (material) operations, which depend on the information operations under study. The non-information effects of such operations vary with the implementation of the causal dependencies between environment changes, further information, and subsequently non-information operations fulfilment. These causal dependencies are examined in article based on technological system example. Technological system is defined as such system which functioning is fully described by operations description in its documentation. According author's opinion, information operations cause changes in non-information (that is material) operations through change of prescriptions. Next, this change leads to material effects change. That is why it is necessary to develop the concept of information and noninformation actions causal dependencies and the concept of effects formation as a result of such causal dependencies [10]. It is the development of the concept of such dependencies that causes conceptual difficulties and thus further difficulties with mathematical modelling of effects formation due to IT use. That is why further research is directed to the description of the causal dependencies between information and non-information (material actions and between information and non-information effects. Such dependencies description further allows analytical predictive 
description of IT use effects using mathematical models. The predictive mathematical models developed of the formation of usage effects of information operations, including noninformation (material) effects that are changed as a result of the information effects. Such model's objective is analytical evaluation of the information operations use operational properties depending on operations use characteristics. The models are also used to evaluate other operational properties, especially the system potential. System potential measured considering the necessary use of information operations. The results presented in this study are aimed at bridging the gap between the need to solve research problems of operational properties regarding IT use based on predictive mathematical models [11-14] and the lack of the necessary concepts and methodology for solving such problems as mathematical problems of estimation, analysis, and planning by operational properties [15-22] with regard of IT use.

\section{CONCEPTUALIZING THE USE OF INFORMATION TECHNOLOGIES}

The use of IT is illustrated in such complex systems that the operation of these systems is technological by its nature. That means that operations of such system are completely specified by descriptions of technological operations in the technical documentation for a complex technological system (hereinafter CTS). According this assumption about the technological nature of functioning, not any action in the system is considered but only technological operations. It further allows asserting that the states of the beginning of CTS operations, the modes of performed technological operations, and the possible resulting states of such operations performance are fully described in the technical documentation for the CTS. The essence of changes in material (non-information) operations as a result of the information operations is that different states of the system and the environment can be implemented, and different requirements can be accommodated by environment. These states and requirements can lead to necessity of CTS to react and to change course of its functioning. For such change information operations required. That is, environment change can lead to changes in information operations. Subsequently, information operations can cause changes in other operations. It is assumed that the number of such changes in operations is finite and can be described by modes of operations. As a result, CTS reacts on environment changes with use of information operations, becoming like "living" system. Effects of system functioning which is "alive" differ depends on information operations characteristics - i.e. depends on how it is "living". How it is "living" is described by finite number of modes of operations in their chains. Such chains depend on environment impact.

Finite number of modes of technological operations specified in the technical documentation of CTS and thus, can be expressed with model which describes chains of operations depending environment impact. Accordingly, knowing the possible changes in the environment and caused by environment impact modes of information operations we can build a model of the possible CTS states as a result of the chains of environment impacts and further information operations. These chains lead to different prescriptions to perform material (non-information) operations and consequently, to different modes of the implementation of these material operations. Various modes of such material operations result in changed effects. These changes cause change in effects compliance to effect requirements of the changing environment. The difference in effects compliance to changing environment demands, depending kind of IT used to realize needed compliance may serve as IT use performance indicator.

So, information operations in the CTS effects are making CTS "living" system. That means information operations allow carrying out material actions in the changing environment with modes of operations that are differ from those which was planned before environment change and its impact on CTS. These modes are better adapted to changing environment conditions and may provide better results. Because of suggestion that number of possible environment states is limited, as well as number of operation modes, it is further asserted that the possible number of chains of information and caused by them material operations is finite as well. As a result, we can model these chains with use of finite discrete algebraic model, for example - graph theoretic one. Before building the algebraic model of information operations use and therefore the use of IT describing these operations, it is necessary to perform conceptual modelling of possible finite sequences of environment states and then caused by them information and material operations. In sequences of such operations and caused by them states there can be alternative relationships and states [23-29] caused by various types of alternatives. It is further assumed that such alternatives are known, their number is finite and that a measure for the possibility of such alternatives to be realized known. As a result, to model possible sequences of relations between states, information and non-information actions, and their subsequent formalization, labelled finite graphs can be used.

\section{FORMALIZING USE OF INFORMATION OPERATIONS AND CORRESPONDING TECHNOLOGIES}

The method of formalization consists of assigning the main concepts and relations between them to graph theoretical objects. To implement such formalization, the graph-theoretic model of the main concepts of the problem is used. Such model was constructed with use of knowledge representation software. It is proposed to use constructed model to instantiate graph theoretic objects - i.e. vertices and arcs. Conceptual model serves as meta-model $\mathrm{f}$ graph theoretic objects. Conceptual model than makes it possible to correctly instantiate the parametric models by parameterization of the graph-theoretic model according conceptual model elements mapping with database records. Then functional models can be constructed by specifying and explicating the functional dependencies between the explicated parameters and variables. As a result, labelled graph theoretic model built, which imitate possible chains of states and operations in environment and system while traversing the graph theoretic model performed and computation of functional dependencies realized wile traversing graph theoretic model. As a result, effects characteristics computed on graph theoretic model. Using these characteristics of effects problems of evaluation, analysis, and synthesis by operational properties, mainly system potential indicators, would be solved. Provided formalization of the dynamic capability or system potential research problems can be represented as construction of graphs and their labels based on conceptual model. 
First, conceptual models built. Next, algebraic graphs are constructed that instantiated with use of conceptual model. Then the graphs are labeled by variables and parameters. Next graph theoretical models are built in which functional dependency labels are assigned to its elements. Finally, functional dependency labels explicated using programming language text. The graph-theoretic models created allow imitating real functioning in real circumstances and given IT used with sequences of computations. This property of the models enables imitation by analytical computations because of possible sequences of states and computations performed by computer are linearized in the sequences which imitate causal relationships of states and operations during real functioning.

Such graph-theoretic models must be connected without loops. In this case they can be traversed to calculate all the required expressions based on functions specified as the labels. The calculations can be performed by traversing the graph in depth or in width with the calculation of the functional relations. Based on labelled graph-theoretic models suggested it is possible to generate a functional and program language code models which describe a finite sequence of functional expressions for the calculation of the system's potential indicators depending on the variables of the problem to be solved.

\section{FORMAL MODEL EXAMPLE OF TECHNOLOGICAL SYSTEM FUNCTIONING WITH IT USE}

An algebraic structural model of the CTS functioning describes the elements and structure of the system workplaces (WP): Let us denote: $e_{j k}-k-$ th element on $j-$ th WP, according to the technical documentation; $e_{j k} \in E_{j}$, where $E_{j}$ - workplace $j=\overline{1, J}$; Realizations of states of WP in appropriate sequences, corresponding to real functioning fulfilled according to the created concept model. At a given moment $t$, part or all WP are functioning. If WP functions than technological operation (TlOp) is performed with it. TlOp implemented on the WP according to one of the possible modes. Mode of operation can be predefined or set by information operation. TlOp can begin only if the specified state of the WP is reached and mode of operation description is part of such state. TlOp can lead to different states as a result of TlOp realization, depending on the environment conditions and mode of operation performed. The set of states of $E_{j}-$ th WP at each moment forms a state of the CTS as a whole:

$$
Q(t)=\bigcup_{j=\overline{1, J}} Q_{E_{j}}(t) .
$$

These system states $Q(t)$ for the moment $t$ are checked at the boundary of the system with its environment. A mathematical model of states at the CTS boundary is built as an algebraic model of CTS states sequences on the CTS boundary and transitions between such states. It is assumed that the number of such states on the boundary is finite. These states on the boundary shall comply with states demanded by environment so these states are checked for compliance. The algebraic model can be represented as a tree. Then, from the constructed algebraic model, a functional model of states of the CTS and its environment as well as their compliance is generated. This model unites the model of states at the boundary of the CTS and the model of the environment states. It is the model which needed in order to calculate CTS potential indicators.

It is further assumed that the number of states at the boundary of the CTS as well as states demanded by its environment is finite. Thus, transitions between states number is finite either. Information operations are needed to check states at the boundary. The results of these information operations are a compliance measure of the CTS and the environment states. The model of such sequence of information operations on the border can be used to determine the CTS potential indicators.

Classes of relations between CTS states modeled as arcs, hyper arcs, and nested graphs at the tree of states. Transitions are a special class of relations that are associated with the mode of operations in this tree. Other classes of relations are joint realizations relation of states (simultaneity relation) and relations of possible transitions between states. The first class arises from the possible implementation of TlOp on several WP at the same time. The second class of relations emerges by the completion of TlOp and next, to one of the alternative states of TlOp termination due to one of possible environment impacts. Some relations classes require input (initial) and output (final) states of different kind (information, non-information). Each of the possible sequences of states and transitions checked on the boundary of the CTS for compliance with environment states. Verification operation is information operation and it is part of a branch of the tree. It is assumed that the number of such sequences, that is - number of tree branches is finite. Let us denote this number as $L$. So, the number of elements of the set of all possible sequences of CTS states is $L$ :

$$
C^{C T C}: \quad\left|C^{C T C}\right|=L .
$$

The sequence of states is assumed as such that for each state before verification and for each state of environment correspond different mode of implementing technological information operation (TIO) of verification. The mode of TIO depends on the state before the start of the TIO. This dependence realized according IT used and the plan of operations. If the state before the start of the TIO, the information technology, and the plan of operations defined, then the mode of TIO defined too. The mode to execute the TIO for verification states on the border of the environment may depend on the environment state changes. Modes of environment operations, which lead to environment states, are not known for sure, but the state sequences, resulting from these operations, transitions between environment states as well as the measure of the possibility of implemented transitions are known. So, as a result of a sequence of environment state transitions and as a result of caused by each sequence modes of implementation of the CTS operations, we can get a pair of states on the boundary. In each pair one state is state of environment, another one is state of CTS and their compliance can be measured. As well possibility of actualization of each pair can be obtained. For this reason, note that in the sequences of states described each pair of states on the boundary corresponds to different branches of the tree of environment states and the tree of CTS states. That branches actualization possibilities can be used to estimate possibility (probability) of appropriate pair of states actualization. 
Let's fix the sequence of environment states (and so transitions between states). For this it is assumed that the actions and states of the environment do not depend on the CTS operations. Opposite, it is assumed that the CTS states depend on the sequence of environment states. As a result of such assumptions, the specified sequences of the environment states can be considered without environment states interrelations with CTS states. That is why the sequences of environment states can be modelled as a tree of possible sequences of environment states. This tree is constructed before a tree of CTS states. In the tree constructed, edges correspond to the transitions of environment states that happen due to possibly not known modes of actions in the environment. The states considered are the states of the environment on the border of the environment with the CTS. Let us denote the number of such sequences of the states of the environment as $M$.

Next, let us denote set of possible sequences of environment states as a result of some modes of environment actions as $C^{C p}$. Thus, $\left|C^{C p}\right|=M$ and the elements $c_{m}^{C p} \in C^{C p}$ are modeled with the branches of the tree of environment states, $m=\overline{1, M}$. Next functional model of the environment is constructed. To construct it, sequences $c_{m}^{C p} \in C^{C p}$, associated with the branches parameterized first. Parameterization performed for states, then for state transitions and then a parameterization of sequences of states performed. The parameterization includes assignment of probabilities of states as well as transition actualizations probabilities. Further, functional dependencies are assigned to transitions. Such dependencies associated with transitions and connect the parameters, the measures of the probability of the states and the dependent variables of the states. A mathematical model of the environment under the assumption of independence of the activities of the environment from CTS states allows further constructing a mathematical model of CTS under given environment changes and then - environment states compliance model. Such compliance is measured on the boundary of environment and CTS by relating corresponding states with use of the appropriate TIO of states compliance measure verification on the boundary. Compliance relations are determined between the nodes of the CTS states tree and the nodes of the environment states tree. The state of the CTS during its functioning depends on the states of the environment and such a dependency in the study of the potential cannot be neglected. That is why modes of implementation of the verification TIO on the boundary of the CTS are associated with one and only one branch of the tree of possible states of the environment. The model of the CTS and its compliance with environment states demands can be constructed as a result. It allows computing the CTS dynamic capability and potential indicators.

Thus, branches of the CTS state tree are constructed under the condition that the branch $c_{m}^{C p} \in C^{C p}$ of the environment tree is given, that is $\left|C^{C T C}\left(c_{m}^{C p}\right)\right|=L_{m}$. Let us assume that the branch $l \in \overline{1, L}$ is built for $c_{m}^{C p} \in C^{C p}$, i.e. $l_{m} \in \overline{1, L_{m}}$. This means that a relationship is defined between $c_{m}^{C p} \in C^{C p}$ and the corresponding it $C^{C T C}\left(c_{m}^{C p}\right)$. Thus, a new tree can be constructed that includes a branch $c_{m}^{C p} \in C^{C p}$ before the root of the $C^{C T C}\left(c_{m}^{C p}\right)$ tree. The resulting model is one which corresponds to all branches. It is used to create the functional model of CTS and then to create the model needed to calculate the CTS potential indicators. It is assumed that the number of states in the state tree branch $l \in \overline{1, L}$ can be variable. It is because the number of operations and so - transitions and accordingly, the number of resulting states could vary. It varies because of the impact of the environment. As well, even under the same environment impact, the duration of the state transitions and the duration of the sequences of actions on different WP vary as well. So, the number of required state verifications at the system and environment boundaries may vary. Let us designate the number of these states as $Q_{l}$ for a given branch $l \in \overline{1, L}$ of the given tree. Each state on the CTS border $q_{l} \in \overline{1, Q_{l}}$ verification corresponds to the implementation of the verification TIO by the specified mode. So, the state corresponding to this mode is $q_{l} \in \overline{1, Q_{l}}$. Any of the state sequence:

$$
\hat{S}_{l . q}=<\hat{y}_{1 . l . q} \ldots \hat{y}_{k . l . q} \ldots \hat{y}_{K . l . q}>\text {, }
$$

verified at the boundary of the CTS and its environment is fully described by the effects of functioning. Effects are determined for the time the state check starts. ${ }^{\wedge}-$ symbol of randomness [31]. This state is compared with the environment state, which specifies the requirements values:

$$
S_{l . q}^{\partial}=<y_{1 . l . q}^{\partial} \ldots y_{k . l . q}^{\partial} \ldots y_{K . l . q}^{\partial}>\text {. }
$$

Then, a probability or possibility measure $P_{l . q}$ of the state's $\hat{S}_{l . q}$ compliance with the requirements $S_{l . q}^{\partial}$ of the environment can be defined as:

$$
\begin{aligned}
& P_{l . q} \\
& =P\left(\hat{A}_{l . q}\right) \\
& =P( \\
& <\hat{y}_{1 . l . q} r_{1} y_{1 . l . q}^{\partial} \ldots \\
& \left.\quad \ldots \hat{y}_{k . l . q} r_{k} y_{k . l . q}^{\partial} \ldots \hat{y}_{K . l . q} r_{K} y_{K . l . q}^{\partial}>\right),
\end{aligned}
$$

where $r_{k}$ is the required relationship between the predicted values of the effect's characteristics and their required values (e.g. <,>) according demands of the environment. The probability or possibility measure of compliance is calculated using a functional model of states compliance on the boundary of the CTS and the environment.

$P\left(\hat{A}_{l . q}\right)-$ the probability or possibility of an event $\hat{A}_{l . q}$. This event consists of this: when verification of the state $\hat{S}_{l . q}$ done for one of the possible branches of the tree, performing a single verification TIO by the defined mode, the required by the environment characteristics of the effects will be achieved by CTS. This event means that the result of the verification TIO is the required intermediate goal of the CTS functioning achieved. The measure of compliance for the implementation of the entire sequence of verification TIO $c_{m}^{C p} \in C^{C p}$ can be than constructed. It is the corresponding measure of compliance for the whole branch of $C^{C T C}\left(c_{m}^{C p}\right)$ and it can be calculated as the probability of a complex event i.e. $-\hat{A}_{l}$. This event is that all the intermediate goals are achieved in the given environment conditions. Each event $\hat{A}_{l}$ probability is:

$$
P\left(\hat{A}_{l}\right)=P\left(\bigcup_{q \in \overline{1, Q_{l}}} \hat{A}_{l . q}\right) .
$$


With the assumption that the probabilities of states compliance for each of the verification TIO are conditionally independent in their sequences:

$$
P\left(\hat{A}_{l}\right)=\prod_{q \in \overline{1, Q_{l}}} \hat{A}_{l . q} .
$$

Let the probability of $\hat{B}_{q, p}$, consisting of the event that transition $a_{q, p}$ will be executed:

$$
\hat{B}_{q, p}=\left(\hat{S}_{l . q}, \hat{S} q, p \overline{1, Q_{l}} \text { l.p },\right.
$$

be equal to $P_{q, p}=P\left(\widehat{B}_{q, p}\right) \sim a_{q, p}$. That means that the probability $P\left(\hat{B}_{q, p}\right)$ is associated with the transition $a_{q, p}$. Then the probability of a branch implementation $v_{l}: l \in \overline{1, L}$ of the tree $C^{C T C}\left(c_{m}^{C p}\right)$ is:

$$
P_{l}=P\left(\bigcap_{a_{q, p} \in v_{l}} \hat{B}_{q, p}\right) .
$$

In the case that these events of transition executions are conditionally independent too:

$$
P_{l}=\prod_{a_{q, p} \in v_{l}} P\left(\widehat{B}_{q, p}\right) .
$$

So, as a scalar indicator of the CTS potential $\psi$ or dynamic capability indicator under condition of given IT use, we can take the expected probability or possibility that the following event will happen: whatever branch $c_{m}^{C p} \in C^{C p}$ and corresponding branches of $C^{C T C}\left(c_{m}^{C p}\right)$ are implemented, there will be compliance between the expected (CTS) and required (by environment) states. As well, this means that whatever changes in the environment happen, needed corresponding to them information and then, material operations will conduct according given IT, and as a result - changing goals of the CTS in conditions of changing environment will be achieved:

$$
\bar{\psi}=P(\hat{C}) \approx \sum_{l \in \overline{1, L}}\left(P_{l} \cdot P\left(\hat{A}_{l}\right)\right) .
$$

Generally, the probability $P(\hat{C})$ of a specified event can be represented as a random variable $\hat{\psi}$, not its expected value $\bar{\psi} . \hat{\psi}$ discrete distribution $f_{\widehat{\psi}}(l)$ could be described by the following vector of pairs:

$$
f_{\widehat{\psi}}(l)=\left(P_{l}, P\left(\hat{A}_{l}\right)\right)
$$

This vector of pairs can be used as the CTS potential indicator in vector form:

$$
\Psi=<f_{\widehat{\psi}}(l), l=\overline{1, L}>.
$$

Indicators presented above describe the different characteristics of the CTS potential or dynamic capability, given that the functioning of the CTS is terminated. But analogously, system potential indicators can be constructed for any moment during functioning. Various CTS potential indicators can be used, for example, in case of criteria of optimism or pessimism use. Such indicators make sense of the various characteristics of the probabilistic measure of compliance of the predicted effects characteristics (CTS) with the requirements for them (caused by environment in different circumstances). With this compliance measured at the boundary of the CTS and its environment at different time the appropriate changes in the CTS that are caused by the environment changes can be measured. The mathematical model of such compliance on the boundary is the basis of further construction of the mathematical model of the CTS potential and dynamic capability estimation and then analysis and synthesis by system potential indicators. To obtain a mathematical model of the system potential estimation based on the specified model, it is necessary to construct models that reveal the values: (2) and (3) with parametric and then functional graph-theoretic models use. Such model building can be interpreted as a special kind of recursive graph extension. Such extension may be conducted until graph constructed, which describes the calculation of the functioning effects on graphs.

Under the extension of graph-theoretic models it is understood a construction operations sequence on these models, which produce required graph. Required graph is such graph that graph models elements, which associated with parameter or variable, are calculated based on the traverse of the graph model created. With the use of the proposed graph-theoretic models in the form of hierarchical trees the properties associated with the elements of the trees are described by replacing the node of the original tree with a composite tree. Functional dependencies on the trees must be specified in such a way that by traversing models specified and by computations of functional dependencies during traverse it will be possible to calculate the values of indicators. Then digitalization success indicators can be estimated as the measure of distance between system potential indicators values before and after the digitalization conducted.

\section{CONCLUSION}

The results obtained enable the evaluation of the predicted values of the system potential and dynamic capabilities indicators depends on characteristics of IT used. As a result, digitalization success indicators can be estimated in the form of distance measure between indicators value before and after digitalization. Analytical estimation of such indicators is possible depending on the variables and options in the mathematical problems to be solved. This shall allow a solution of contemporary problems of digitalization research using predictive analytical mathematical models and using of known mathematical methods of such mathematical problems research. Examples of such research problems are problems related to modern (i.e. - digital) IT use performance, efficiency, effectiveness. Possible applications include choosing the best digital technologies characteristics, choosing IT and TIO characteristics for the optimal implementation of digital IT, choosing best digitalization scenarios. It makes it possible, as a result, to overcome the existing gap between the need to solve research problems in digitalization performance, efficiency, effectiveness based on mathematical models and methods and the lack of the necessary concepts and methodology for solving such problems.

\section{ACKNOWLEDGMENT}

The reported study was funded by RFBR according to the research project № 19-08-00989 A.

\section{REFERENCES}

[1] D. Teece, G. Pisano and A. Shuen. "Dynamics capabilities and strategic management," Strateg. Manag. J., vol. 18, p.p, 509-533, 1997.

[2] C. Wang, P. Ahmed. "Dynamic capabilities: A review and research agenda,” Int. J. Manag. Rev., vol. 9, p.p. 31-51, 2007. 
[3] D. Teece. "Explicating dynamic capabilities: The nature and foundations of (sustainable) enterprise performance," Strateg. Manag. J., vol. 28, p.p. 1319-1350, 2007.

[4] A. Ashimov, A. Geyda, I. Lysenko and R. Yusupov. "System Functioning Efficiency and Other System Operational Properties: Research Problems, Evaluation Method," SPIIRAS Proceedings, vol.5(60), p.p. 241-270, 2018.

[5] A. Geyda, I. Lysenko. "Operational properties of agile systems and their functioning investigation problems: Conceptual aspects," J Appl. Inform, vol.12, p.p. 93-106, 2017.

[6] A. Geyda, I. Lysenko. "Schemas for the analytical estimation of the operational properties of agile systems," SHS Web Conf. 2017; 35 , 01058.

[7] A. Geyda, I. Lysenko and R. Yusupov. "Main concepts and principles for information technologies operational properties research," Spiiran Proc., vol. 42, 5-36, 2015.

[8] A. Geyda, Z. Ismailova, I. Klitny and I. Lysenko. "Research problems in operating and exchange properties of systems". Spiiran Proc., vol. 35, p.p. 136-160, 2014.

[9] A. Geyda. "Dynamic Capabilities Indicators Estimation of Information Technology Usage in Technological Systems," In: Dolinina O., Brovko A., Pechenkin V., Lvov A., Zhmud V., Kreinovich V. (eds) Recent Research in Control Engineering and Decision Making. (Studies in Systems, Decision and Control, No 199), p.p.379-395, Springer, 2019

[10] A. Geyda, I. Lysenko. "Modeling of Information Operations Effects: Technological Systems Example" Future Internet, vol. 11:62, 2019.

[11] J. Taylor. Decision Management Systems: A Practical Guide to Using Business Rules and Predictive Analytics, IBM Press, Indianapolis, IN, USA, 312p., 2011.

[12] T. Kendrick. How to Manage Complex Programs, AMACOM, New York, NY, USA, 336p., 2016.

[13] T. Dinsmore. Disruptive Analytics: Charting Your Strategy for NextGeneration Business Analytics, Apress, New York, NY, USA, 276p, 2016.

[14] A. Downey. Think Complexity. Complexity Science and Computational Modeling, O'Reilly Media, Sebastopol, CA, USA, 160p., 2012.

[15] Cokins, G. Performance Management: Myth or Reality? In: Performance Management: Integrating Strategy Execution, Methodologies, Risk, and Analytics; Wiley: Hoboken, NJ, USA, 2009; 274p.

[16] G. Cokins. "Why is Modeling Foundational to Performance Management?" Dashboard Inside Newsletter, March 2009.
[17] C. Hood, S. Wiedemann, S. Fichtinger and U. Pautz. "Requirements Management," In: The Interface between Requirements Development and All Other Systems Engineering Processes, Springer: New York, NY, USA, 275p., 2008.

[18] D. Hybertson. Model-Oriented Systems Engineering Science: A Unifying Framework for Traditional and Complex Systems; Auerbach: Berlin, Germany, 379p., 2009.

[19] E. Aslaksen. The System Concept and Its Application to Engineering; Springer: New York, NY, USA, 266p., 2013

[20] E. Aslaksen. Designing Complex Systems. Foundations of Design in the Functional Domain; Complex and Enterprise Systems Engineering Series; CRC Press, Boca Raton, FL, USA, 176p., 2008.

[21] F. Franceschini, M. Galetto and D. Maisano. Management by Measurement: Designing Key Indicators and Performance Measurement Systems, Springer, New York, NY, USA, 242p., 2007.

[22] G. Roedler, R. Schimmoller, D. Rhodes, and C. Jones. Systems Engineering Leading Indicators Guide, INCOSE Technical Product, INCOSE-TP-2005-001-03. Version 2.0; Massachusetts Institute of Technology, INCOSE, PSM, Cambridge, MA, USA, 146p., 2010.

[23] G. Tanaka. Digital Deflation: The Productivity Revolution and How It Will Ignite the Economy, McGraw-Hill, New York, NY, USA, 418p., 2003.

[24] Guide to the System Engineering Body of Knowledge, SEBoK v. 1.9.1; INCOSE, San Diego, CA, USA, 2018.

[25] J. Simpson, M. Simpson. "Formal Systems Concepts. Formal, theoretical aspects of systems engineering. Comments on Principles of Complex Systems for Systems Engineering," Syst. Eng., vol. 13, p.p. 204-207, 2010.

[26] J. Elm, D. Goldenson, K. Emam, and A. Neisa. "A Survey of Systems Engineering Effectiveness-Initial Results (with Detailed Survey Response Data)," NDIA SE Effectiveness Committee, Special Report CMU/SEI-2008-SR-034, Acquisition Support Program, CarnegieMellon University, Pittsburgh, PA, USA, 288p., 2008.

[27] N. Patel. Organization and Systems Design. Theory of Deferred Action, Palgrave McMillan, New York, NY, USA, 288p., 2006.

[28] R. Stevens. Engineering Mega-Systems: The Challenge of Systems Engineering in the Information Age, Complex and Enterprise Systems Engineering Series, CRC Press, Boca Raton, FL, USA, 256p., 2011.

[29] P. Mikalef, A. Pateli. "Information technology-enabled dynamic capabilities and their indirect effect on competitive performance: Findings from PLS-SEM and fsQCA,” J. Bus. Res., vol 70, p.p. 1-16, 2017 